\title{
Effects of physical exercise on central nervous system functions: a review of brain region specific adaptations
}

\author{
Julie A Morgan ${ }^{1}$, Frances Corrigan ${ }^{2}$ and Bernhard T Baune ${ }^{1 *}$
}

\begin{abstract}
Pathologies of central nervous system (CNS) functions are involved in prevalent conditions such as Alzheimer's disease, depression, and Parkinson's disease. Notable pathologies include dysfunctions of circadian rhythm, central metabolism, cardiovascular function, central stress responses, and movement mediated by the basal ganglia. Although evidence suggests exercise may benefit these conditions, the neurobiological mechanisms of exercise in specific brain regions involved in these important CNS functions have yet to be clarified. Here we review murine evidence about the effects of exercise on discrete brain regions involved in important CNS functions. Exercise effects on circadian rhythm, central metabolism, cardiovascular function, stress responses in the brain stem and hypothalamic pituitary axis, and movement are examined. The databases Pubmed, Web of Science, and Embase were searched for articles investigating regional brain adaptations to exercise. Brain regions examined included the brain stem, hypothalamus, and basal ganglia. We found evidence of multiple regional adaptations to both forced and voluntary exercise. Exercise can induce molecular adaptations in neuronal function in many instances. Taken together, these findings suggest that the regional physiological adaptations that occur with exercise could constitute a promising field for elucidating molecular and cellular mechanisms of recovery in psychiatric and neurological health conditions.
\end{abstract}

Keywords: Exercise, Neurophysiology, Neurobiology, Brain stem, Hypothalamus, Basal nuclei, Disease, Depression, Stress, Neurodegenerative Diseases

\section{Review}

\section{Introduction}

Health can be disrupted by stress of acute or chronic duration, and may be either physiological or psychological [1]. Extreme stressors that elicit the acute 'fight or flight' responses, such as violence or natural disasters can and do occur however, the chronic low level daily hassles or issues that cause stress and result in sleep loss, comfort eating with resultant weight gain, and smoking or excessive drinking of alcohol are more common [2]. Chronic stress is considered to contribute to the aetiology of a range of psychiatric and neurological conditions including depression and Alzheimer's disease [3,4]. Furthermore, these conditions often involve the dysregulation

\footnotetext{
* Correspondence: Bernhard.baune@adelaide.edu.au

'University of Adelaide, School of Medicine, Discipline of Psychiatry, Psychiatric Neuroscience Laboratory, Adelaide, South Australia, Australia Full list of author information is available at the end of the article
}

of important functions coordinated by the brain such as circadian rhythms [5,6], central metabolic function $[7,8]$, and stress responses via the hypothalamic pituitary adrenal axis (HPA) $[9,10]$.

Basic science and clinical research is providing promising evidence of physical exercise-induced outcomes for several prevalent neurological and psychiatric conditions (CNS). This occurs in part through increases in neurotrophic factors such as brain derived neurotrophic factor (BDNF) [3,11,12], reductions in oxidative stress [13] and limiting neuroinflammation $[14,15]$. However many of the mechanisms by which exercise exerts its effects in the brain remain largely unknown.

Nevertheless, a substantial body of literature has now investigated the effects of exercise in a range of populations [16-18], and brain regions such as the hippocampus [19-21], resulting in advancement in the understanding of the exercise on a number of areas including cognitive 
functioning and the neurobiology of learning and memory. However, considerably less work has investigated the impacts of exercise on more primitive brain regions including the brainstem, hypothalamus, and basal ganglia, which are involved in other important functions for health. These include the regulation of diurnal rhythm and circadian function, food intake, cardiovascular function, and responses to stressors. There is increasing recognition of metabolic dysfunction in Alzheimer's disease [22] and depression [23]. Moreover, there is growing evidence that brain metabolic disturbances such as central insulin resistance are involved in the pathogenesis and progression of Alzheimer's disease [22], and that circadian rhythm and HPA axis disturbances can be evident in depression and Alzheimer's disease [5,6,10]. Given the roles of these CNS dysfunctions in the aetiology and progression of these conditions, understanding the regional neurobiology of such mechanisms seems critical for advancing preventative measures and treatments. The aim of this review is therefore to elucidate and critically evaluate the effects of chronic exercise in the context of basic drive functions in the brainstem, hypothalamus, pituitary gland and basal ganglia. Particular focus will be on the exerciseinduced regulatory effects on energy balance and metabolism, cardiovascular regulation, circadian function, and responses to stress.

\section{Materials and methods}

The PRISMA guidelines (Preferred Reporting Items for Systematic Reviews and Meta-analysis) for reporting systematic reviews and meta-analyses checklist items were followed in the reporting of this review (for the items eligibility criteria; information sources; search; and study selection) [24].

\section{Searches}

Searches were conducted in the electronic databases Pubmed, Embase, Medline, and Web of Science. The search terms exercise; voluntary wheel running, and wheel running were combined using OR, then combined using AND with the terms: brain stem; hypothalamus; paraventricular nucleus; suprachiasmic nucleus; ventromedial nucleus; thalamus; basal nuclei; neurobiology; energy; metabolism; metabolic; autophagy; circadian; diurnal; cardiovascular; sympathetic; parasympathetic; and HPA axis. The 3408 articles returned were screened by review of the titles and abstracts for relevance to the aims of this paper, and contained 222 duplicates. Papers were exported and stored in Endnote X6.0.1 software for further consideration of the full text (see Figure 1).

\section{Inclusion and exclusion criteria}

Articles published in the English language were selected involving murine studies on adult animals. Murine studies investigating chronic exercise-induced effects on central neurobiological functions in the brain stem, hypothalamus, thalamus, and basal ganglia were included. Given that the focus of this review relates to murine neurobiological mechanisms in the brain stem, hypothalamus, thalamus, and basal ganglia, research investigating human participants was excluded. In addition, murine studies investigating the effects of exercise on peripheral and plasma measures; drug interventions; addiction; post traumatic brain injury or spinal cord injury; sexual function and dysfunction; autism spectrum disorders; attention deficit hyperactivity disorder; gene expression; and whole brain analyses without regional brain distinctions were excluded. Other brain regions including those related to the limbic system such as the hippocampus; cortex; amygdala; and prefrontal cortex were excluded because these regions are involved in emotion and cognition generation rather than fundamental physiological processes. Although exerciseinduced physiological interactions between the limbic system and the brain stem, hypothalamus, thalamus, and basal ganglia require investigation, these topics are complex and will require extensive investigation that is beyond the scope of this review. Fifty-seven papers without full text were excluded, and searches were limited to the years 1998 to 2015. The final databases searches were conducted on 3rd February 2015.

\section{Results and discussion}

\section{Voluntary and forced exercise methods}

Several noteworthy points about murine research involving exercising animals require clarification. Murine studies investigating adaptations to exercise utilise a range of methodologies involving varied types of exercise, such as voluntary wheel running (VWR) or forced exercise. They also utilise different intensities of exercise ranging from low intensity, moderate, or high intensity. Mice running voluntarily on a running wheel tend to run intermittently in short bursts and at a preferred cruising speed [25]. However, forced exercise often involves speeds set at a constant rate, for example on a rodent treadmill set at 8 metres/minute, or incrementally increased speeds over the duration of the exercise program. In addition, forced exercise is believed to involve the potential additional component of emotional stress from coercion of the animal, and this makes it difficult to differentiate between the effects of the physical stress of exercise, and the effects of the emotional stress of coercion, thereby potentially confounding the investigated outcomes [12,26] (see Table 1). The voluntary or forced mode of exercise undertaken by animals is therefore an important concern, and is reported throughout this review. 


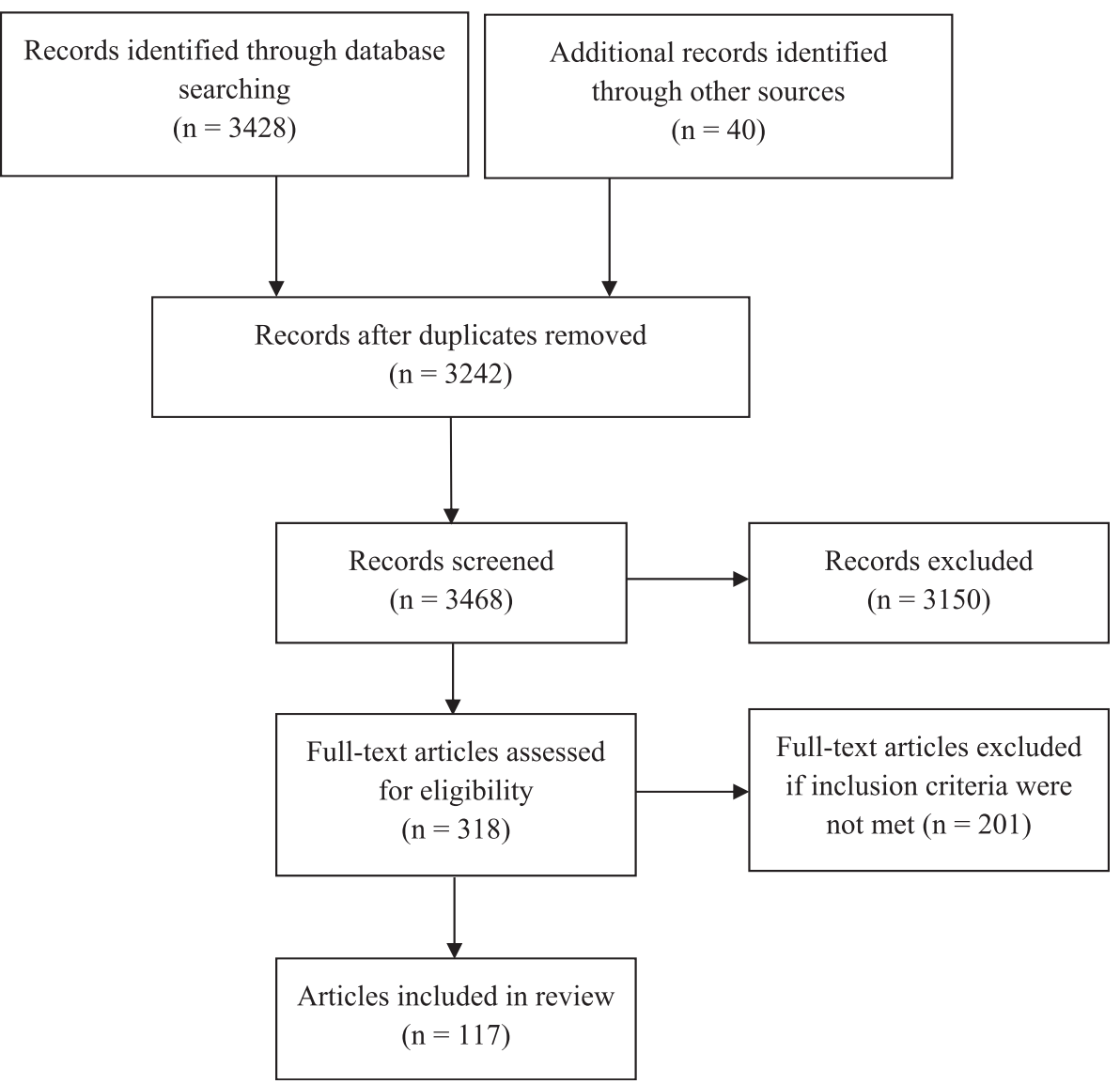

Figure 1 Flow diagram of included studies (adapted from [24]).

Table 1 The differences between voluntary and forced exercise in murine studies

\begin{tabular}{ll}
\hline Voluntary exercise & Forced exercise \\
\hline Self-selected cruising speed & Researcher pre-prescribed speeds \\
Variable speeds & Constant speeds \\
Short bursts of exercise & Relatively long periods of exercise \\
No coercion of the animal & Coercion of the animal \\
No psychological stress arising & Possible psychological stress arising \\
from coercion & from coercion \\
No physiological cascades & Possible physiological cascades \\
arising from & arising from potential psychological \\
psychological stress & stress from coercion \\
from coercion & \\
No potential for physiological & Potential for physiological \\
responses to the psychological & responses to the psychological \\
stress of coercion to interact with & stress of coercion to interact with \\
or confound the parameters & or confound the parameters under \\
under investigation & investigation \\
No potential for confounded & Potential for confounded findings \\
findings due to psychological & due to physiological stress \\
stress physiology & physiology \\
Directly translatable to clinical & Difficulty with translation to \\
studies & clinical studies \\
\hline
\end{tabular}

\section{Brain stem}

Dorsal raphe nucleus adaptations to exercise

The dorsal raphe nucleus contains serotonergic neurons that have extensive projections to many brain regions. These include those involved with mood states and behaviour [27], such as the amygdala, hippocampus and cerebral cortex [28] that are widely implicated in stress, anxiety and depression [29]. VWR has a range of impacts on serotonin-mediated responses to stressors. These include effects on serotonin receptors that when activated, inhibit serotonin synthesis and release, and are thus implicated in resilience to stress and anxiety [30]. Six weeks of VWR reduced the activation of serotonergic neurons in the rostral and mid dorsal and ventral DRN in response to uncontrollable stress, as detected by c-fos staining [31]. This may be a mechanism that contributes to reducing stress responses in rats.

There are also time dependent changes in the receptor mRNA in the DRN. Three weeks or six weeks, but not 3 days of VWR, increased the mean serotonin transporter $(5 \mathrm{HTT})$ mRNA (conducts the reuptake of extracellular serotonin into presynaptic neurons) in the DRN $(\mathrm{p}=0.02)$ [32]. The mean DRN serotonin receptor $1 \mathrm{~A}$ 
$\left(5 \mathrm{HT}_{1 \mathrm{~A}}\right)$ mRNA $(\mathrm{p}=0.05)$ was also increased [32]. In addition, 3 days, 3 weeks and 6 weeks VWR decreased serotonin receptor $1 \mathrm{~B}\left(5 \mathrm{HT}_{1 \mathrm{~B}}\right)$ mRNA in the rostral and mid ventral DRN [32]. Interestingly, transient increases in mean $\alpha_{1 b}$-adrenergic receptor $\left(\alpha_{1 b}\right.$-ADR) mRNA at three weeks had returned to baseline levels at 6 weeks [32]. The temporal aspects of these changes suggest that the duration of VWR is a factor effecting $5 \mathrm{HTT}, 5 \mathrm{HT}_{1 \mathrm{~A}}$ mRNA, $5 \mathrm{HT}_{1 \mathrm{~B}} \mathrm{mRNA}$, and $\alpha_{1 b}$ ADR mRNA in the DRN [33]. Importantly, VWR appears to induce mechanisms that directly affect serotonergic neuron excitability and inhibition in the DRN.

Time dependent adaptations with VWR are also evident in stress induced behavioural parameters mediated by the DRN $[34,35]$. VWR induced reductions in uncontrolled stress exacerbated behavioural deficits in shuttle box escape latencies were evident at 6 weeks but not at 2 weeks [34]. Curiously, 6 weeks of forced wheel running and VWR, but not forced treadmill running were found effective in reducing uncontrollable stress induced deficits in learning [35]. This suggests that exercise involving a forced component may be therapeutic in some instances [35]. In summary, VWR results in time dependent changes in basal levels of $5 \mathrm{HTT}$, autoreceptor $5 \mathrm{HT}_{1 \mathrm{~A}}$ and $5 \mathrm{HT}_{1 \mathrm{~B}}$ mRNA, and $\alpha_{1 b}$-ADR in the DRN in region specific ways. These factors appear to be involved in VWR induced attenuations in uncontrollable stress induced deficits in latencies to escape from shuttle box testing in rats. The effects of exercise on serotonin modulation in the DRN are therefore noteworthy for their positive effects on behavioural responses to stress. Speculatively speaking, if serotonergic modulation occurs in the DRN with VWR, this could mediate the input of serotonergic neurons to regions such as the amygdala and hippocampus and have subsequent effects on limbic and cognitive functions. Moreover, the modulation of serotonin in the DRN [31] also has potentially important implications for serotonergic afferent neuronal pathways linking the suprachiasmic nucleus in the hypothalamus that are involved in circadian rhythm function, and this is addressed later in the review.

\section{Exercise-induced changes in the locus coeruleus}

Noradrenergic neurons in the locus coeruleus (LC) are involved in the regulation of attention, arousal, and vigilance responses to stress [36,37]. Stress responses arising from the LC occur in part through signalling via norepinephrine accompanied by galanin - a regulatory peptide formed from the cleavage of preprogalanin. Galanin attenuates neuronal hyper-excitability and may therefore be involved in the noradrenergic neurons adaptation to stress [38]. Exercise induces a range of effects on galanin and preprogalanin. VWR for 5-6 weeks reduced noradrenalin during and after foot shock stress, although it did not alter mRNA expression of $\mathrm{TH}$ or levels of galanin in the LC [39]. In contrast, three weeks of VWR resulted in significant elevations of galanin in the LC after contextual fear conditioning [40]. The authors attributed this to being due to dose dependent differences in the distances run, with their Long-Evans rats running around 20 times further [40] than the Fischer 344 rats [39]. Later studies attempted to control for the confounding factor of the stressors involved in the fear conditioning paradigm by removing stressful behavioural testing, and found that 3 weeks of VWR increased both preprogalanin and galanin expression in the LC [37,41]. Moreover, increased galanin mRNA has also been demonstrated after 3 weeks of VWR in rats selectively bred for greater aerobic capacity, with a correlation evident between the distance run and galanin expression $(\mathrm{r}-0.317, \mathrm{p}-0.028)$ [42]. Voluntary exercise therefore appears to increase galanin and preprogalanin, with possible correlations between its expression and the distances run. Of particular note, is that the locus coeruleus has excitatory input into activation of the hypothalamic pituitary axis (HPA) responses to acute stressors [43]. Elevations in galanin and preprogalanin could therefore contribute to attenuating locus coeruleus excitatory responses to acute stress, and this may have effects on downstream HPA activation. Further investigation is required to clarify this possibility.

\section{Hypothalamus}

\section{Circadian clock adaptations to exercise}

The hypothalamus has an instrumental role in coordinating visceral and drive functions. Functions of the hypothalamus include maintaining energy balance, metabolism, autonomic nervous system modulation, and the circadian clock. The circadian clock is a timing mechanism that endogenously coordinates biochemical, physiological, and behavioural processes with the 24 hour cycle of light and dark [44]. Circadian functioning deteriorates with ageing, and can be disrupted by chronic stress [45]. Circadian dysfunction is implicated in the progression of neurodegenerative conditions [46] and in depression, possibly through alterations in hormones such as cortisol, norepinephrine and melatonin [6].

There is increasing evidence that exercise has noteworthy effects on sleep/wake cycles and circadian clock modulation in both humans and rodents, although the mechanisms involved are not fully understood. The suprachiasmic nucleus $(\mathrm{SCN})$ is considered to be the central coordinating nucleus of circadian functioning although this occurs with some involvement of the brain stem [47]. In rodents, vigorous voluntary wheel running ad libitum provides feedback to regulate the central circadian clock and scheduled exercise can contribute to entraining circadian behaviour [48]. These adaptations occur in part via VWR related arousal signals that relay from dorsal raphe serotonergic pathways to the SCN $[48,49]$. The voluntary 
and spontaneous movement of mice such as grooming, moving or walking, acutely modulates SCN circadian clock pacemaker activity by reducing the amplitude of SCN electrical activity in a duration and intensitydependant manner [47]. This is consistent with other findings that age related declines in SCN amplitude and rhythmicity in male mice are attenuated with access to a running wheel [50]. VWR increases serotonin in the SCN suggesting that serotonin could be a mechanism that mediates SCN amplitude [49]. Indeed, selective lesion of 5-HT terminals in the SCN prevents VWR induced circadian synchronicity [49]. Finally, VWR induces adaptations in hypothalamic heat shock proteins. Heat shock proteins are families of proteins that have robust cytoprotective properties and act as chaperones for other intracellular protein molecules, thereby contributing to cellular resistance to stressors [51]. VWR for 6 weeks in adult male Fischer rats induced elevated levels of the heat shock protein72 (HSP72) $(\mathrm{p}=0.0019)$ in the context of interleukin 1 beta immune challenge in the hypothalamus, suggesting that VWR induces greater cellular resistance to immune challenge stress in this region [52]. Findings from rodent studies are consistent with results from human trials, and together suggest that exercise has potential for altering aspects of circadian dysfunction [48]. This has encouraging potential for a range of human conditions because poor sleep is thought to be a factor in the aetiology of prevalent mental health conditions including anxiety and depression [53], and may also be involved in the pathophysiology of neurodegenerative conditions such as Alzheimer's disease [5].

\section{Exercise effects food intake and energy balance}

The hypothalamus also contains nuclei involved in maintaining energy balance, including the arcuate nucleus, paraventricular nucleus, and dorsomedial and ventromedial hypothalamus. Energy intake and imbalance are contributing factors in the aetiology of neurodegeneration. More specifically, insulin resistance and diabetes in midlife is a risk factor for Parkinson's disease in later life [54,55], and a poor diet involving high fat intake or metabolic disruption such as metabolic syndrome (MetS) can contribute to disease progression in Alzheimer's disease $[7,8]$. Healthy leptin and insulin signalling in the hypothalamus are central factors in energy balance mechanisms, because reductions in these cascades can result in an increase in food intake and weight gain or obesity [56-58].

Short term VWR impacts on various CNS measures related to energy balance and food intake. VWR for 2 days to 1 week reduces the intake of high-fat chow in Sprague-Dawley and F344 rats $[59,60]$. This occurs via reduced meal size and meal frequency from activation of the corticotropin-releasing factor (CRF/CRH) pathway in the dorsomedullary hypothalamus [59]. A significant increase in leptin signalling in the ventral tegmental area also occurs [60], and these factors suggest VWR has a modulatory effect on food choice through CRF and leptin signalling $[59,60]$. Furthermore, the delivery of an exogenous leptin receptor antagonist in the hypothalamus results in significantly reduced VWR $(\mathrm{p}=0.03)$ in F344-Brown Norway rats [61]. However, forced exercise also has metabolic effects in the hypothalamus. Forced uphill treadmill running for 30 minutes, 4 times weekly, for 8 weeks increased tyrosine phosphorylation of insulin receptor 2 (IRS2) with corresponding elevations in IRS2 and subsequent increases in Akt phosphorylation and insulin signalling in the hypothalamus [58]. However, VWR was not included in this study to ascertain potential differential effects. It is possible that the stress from coercion resulted in altered neurophysiological metabolic responses to exercise, thereby confounding the results. Nevertheless, both VWR and forced exercise may result in beneficial alterations to central metabolic status.

The effects of longer term VWR on weight and metabolic status at different stages of the lifespan were investigated in the Berlin Fat Mouse Inbred stain - bred for its predisposition for the development of obesity and metabolic syndrome. Chronic VWR in this breed resulted in the amelioration of weight gain, body fat mass, daily energy intake, and peripheral features of MetS arising from a high fat diet [62]. Similarly, in a Sprague-Dawley model of mid-older age obesity, 2 weeks of VWR inhibited food intake (nearly $50 \%$ or $\mathrm{p}<0.001$ ) [63]. These reductions occurred with modest amounts of VWR that significantly increased leptin signalling in the ventral tegmental area (VTA) (but not the hypothalamus) with resultant reductions in high fat-diet intake and subsequent weight loss [63]. It is noteworthy that more recent work utilising forced methods suggests that chronic forced treadmill exercise does not sensitise leptin function in the hypothalamus [64]. Further work is therefore required to clarify this inconsistency and the mechanisms involved, and in particular, whether physiological cascades involved with a stress response to forced exercise are involved. Interestingly, starting VWR prior to adulthood (at 3 weeks of age) resulted in reduced food intake, whilst initiating exercise from early adulthood (at 9 weeks) increased food intake, although circulating insulin levels remained within the normal range [62]. VWR may therefore attenuate characteristics of Mets arising from diet related energy imbalances and obesity, and may have age related effects on food intake. Further studies on these topics would provide useful clarification about these factors.

Exercise induces autophagy in peripheral skeletal muscle and cardiac tissue, and is a mechanism that contributes to exercise-induced glucose homeostasis via the BLC2 phosphorylation sites [65]. Autophagy is characterised by lysosomal degradation pathways that transfer materials 
from the cytoplasm to the lysosome. This serves to recycle cellular components such as damaged organelles and aggregated proteins for cellular nutrition during starvation, or to meet higher energy demands [66,67]. Although studies investigating potential exercise-induced autophagy in brain regions has not identified its occurrence in the hypothalamus, exercise increases the transgenic fluorescing autophagy marker GFP-LC3 in the anterior cerebral cortex [68]. The potential for autophagy in the hypothalamus and other brain regions requires further careful investigation, because the authors noted the possibility that different methods of sample preparation might result in more sensitive detection of autophagy markers in other brain regions [67]. This is an important topic for investigation because disruptions of autophagy are implicated in neurodegenerative conditions [68]. Future examinations of exercise-induced autophagy in other brain regions therefore ought to utilise methods that are more sensitive so that the mechanisms involved could be elucidated.

Several other exercise-induced mechanisms in the hypothalamus could contribute more indirectly to energy balance and healthy metabolic function. Excessive lipid mass can result in increased endoplasmic reticulum stress that inhibits liver insulin actions, and is a molecular mechanism that contributes to the onset of type two diabetes [69] and increases the risk of progression in Alzheimer's disease $[7,8]$. In contrast to expectations, three weeks of VWR in mice fed high-fat diets increased levels of endoplasmic reticulum stress (ERS) [70]. The ERS marker ATFF6 was increased for high runners and low runners, while eIF2 $\alpha$ was increased in high runners only [70]. The metabolic effects of exercise therefore appear not be related to exercise-induced reductions in ERS. However, it is also possible that physiological mechanisms involved with ERS due to exercise differ from those resulting from a high fat diet, and further research could aid in clarifying this issue. Additional effects of exercise are evident in mitochondria, that produce more than $90 \%$ of cellular energy [71] required for undertaking cellular functions (for further review see [72]). Moreover, in 8 week old mice, eight weeks of forced treadmill running (6 days/week at $25 \mathrm{metres} / \mathrm{min}$ with $5 \%$ incline) significantly increases the mitochondrial DNA (mtDNA) copy number relative to nuclear DNA in the hypothalamus [73]. A limitation of this study is that there is no examination of comparisons with older mice or the use of VWR to determine potential differences evident due to these factors. Nonetheless, a relatively demanding treadmill running protocol can contribute to improving hypothalamic cellular energy dysfunction.

\section{Exercise-induced cardiovascular system changes}

It is now widely recognised that chronic regular exercise has an important role in cardiovascular health [74] although the neurophysiological mechanisms responsible for cardiovascular function are less well understood. The medullary nucleus tractus solitarii (NTS) is thought to have a fundamental role in coordinating complex adaptations to exercise through communication with the hypothalamus [75]. Chronic exercise related adaptations to the CNS kallikrein-kinin system might contribute to this function. The kallikrein-kinin system generates peptides involved in sodium regulation, blood pressure, and inflammation [76]. The activation of B2 kinin receptors, a mediator of the effects of kinins in this system, are involved in the modulation of cardiovascular responses to stress [77]. In hypertensive rats, as in humans, central kinin B2 receptor density is higher in several brain regions including the medullary nuclei [77]. Ten weeks of treadmill exercise at $50-70 \%$ V02max in male Wistar rats increased specific $\mathrm{B} 2$ receptor binding sites in the paratrigeminal nucleus and nucleus solitarii, as well asincreased receptor density in the medulla [77]. This suggests enhancement of the kallikrein-kinin system function may modulate the cardiovascular responses to exercise or stress [77]. However, treadmill running also affects mechanisms relating to autonomic function. Treadmill running for 3 months at 50-60\% Vo2 max, significantly increased oxytocin mRNA levels in the commissural NTS in male normotensive rats, which was associated with increased autonomic cardiac function [78]. However, these mechanisms require investigation using VWR protocols due to potential confounding factors from forced exercise. Nonetheless, exercise-induced effects from VWR are also evident. VWR for 50 days resulted in dendritic plasticity seen as reduced dendritic intersecting per dendritic field in exercised rats compared to sedentary controls in the NTS, posterior hypothalamus, periaquaductal gray, rostral ventrolateral medulla and nucleus cuneatus [79]. Of note is that the dendritic plasticity was related to peak physical performance [79]. These results are pertinent, as it has been suggested that greater dendritic branching (in the RVLM) may contribute to greater sensitivity in these neurons that mediate excitatory responses, thereby contributing to the pathogenesis of cardiovascular disease [80].

Regulation of the autonomic nervous system is also critical to central cardiovascular function, and forced exercise may contribute to the modulation of these systems. In rodents, forced protocols have demonstrated modulation of central cardiovascular neural controls, leading to modified resting cardiovascular parameters such as mean arterial pressure, and heart rate, and reduced sympathetic nervous system (SNS) activity [81-85]. These adaptations occurred through enhanced basal GABAergic function via increased neural nitric oxide synthase (nNOS), that inhibits sympathetic outflow from the paraventricular nucleus (PVN)); GAD67 (which converts glutamine into the inhibitory neurotransmitter GABA); and gephyrin (a component of inhibitory synapses in the anterior and posterior 
hypothalamus) [81]. Nevertheless, the use of voluntary running methods to eliminate the potential for physiological (psychological stress related) confounding factors is likely to provide more sound and translatable results.

\section{HPA axis adaptations with exercise}

Hypothalamic-pituitary adrenal axis (HPA) activation occurs with both psychological and physiological stressors. Excitatory signals from the amygdala, PFC and hippocampus to the PVN of the hypothalamus stimulate the release of CRH. This thenactivates the secretion of adrenocorticotropic hormone (ACTH) from the pituitary into circulation, resulting in the release of glucocorticoids (GCs) (cortisol in humans and corticosterone in rodents) from the adrenal cortex [86]. GCs then modulate and control the stress response, exerting a diverse range of effects on a wide variety of physiological systems including metabolism and immunity. Moreover, GCs, via binding to the glucocorticoid receptor (GR) inhibit the further release of $\mathrm{CRH}$, thereby switching off the release of further GCs [87]. Dysfunction of the HPA axis in patients with major depression is one of the most consistent findings in biological psychiatry. Patients with depression have increased plasma and CSF concentrations of GCs, an exaggerated $\mathrm{GC}$ response to $\mathrm{ACTH}$, and also appear to have dysregulation of the inhibitory feedback of GCs [88]. HPA axis responses are therefore a critical dimension of the treatment of these conditions.

The HPA axis response to voluntary exercise occurs as outlined above. It is a normal adaptive mechanism in response to the increased energy requirements of peripheral tissues, and is a physiological stressor without the psychological stress of fear [86] (unless exercise is forced when this potential is present). Chronic VWR has demonstrated effects on HPA axis parameters in rodents, including increased size and mass of the right adrenal medulla, adaptive changes in ACTH levels [89], and the normalisation of GC levels [90,91]. There is also evidence that VWR can attenuate the HPA axis response to psychological stressors. VWR has been demonstrated to attenuate rises in plasma ACTH arising from foot-shock and cage-switch stressors [92]. Moreover, 6 weeks of VWR in male Sprague-Dawley rats attenuated HPA axis responses to low intensity stressors, such as exposure to a novel environment, 85 decibel $(\mathrm{dB})$ noise, and this was more successful than 1 or 3 weeks of VWR [93]. These results are consistent with other work demonstrating greater habituation to noise stressors with VWR $[94,95]$. It should be noted though that other studies have found no changes in plasma ACTH with VWR after repeated foot-shock [92]. These inconsistencies could be due to the varied stressors involved, however, additional research would clarify this hypothesis.
In the hypothalamus, research has investigated VWR induced adaptations in HPA axis parameters including CRH mRNA, c-fos, arginine vasopressin, and CRH receptor 1 mRNA [93,96]. Six weeks of ad libitum wheel running reduced CRH mRNA in the hypothalamus in the context of repeated noise stressors; and both ad libitum and intermittent (24 hours out of 72) access to VWR resulted in a significant reduction of $c$-fos expression in the paraventricular nucleus of the hypothalamus [93]. No changes however, were found in arginine vasopressin or CRH receptor $1 \mathrm{mRNA}$ in the paraventricular nucleus [96]. Reduced c-fos expression in the PVN with voluntary and intermittent wheel running could suggest attenuated activation of the PVN neurons that may contribute to reduced excitatory input from the PVN to the pituitary, potentially resulting in a reduction in the release of ACTH. Moreover, it is encouraging that relatively reduced (intermittent) access to VWR can have positive effects on PVN $c$-fos expression.

The findings of exercise-induced changes in central parameters of the HPA axis may be obscured when forced exercise methods such as treadmill running are used. For example, one study investigating the effects of incrementally increasing forced swimming for 6 weeks found decreases in hypothalamic glucocorticoid receptor mRNA $(\mathrm{p}<0.01)$ from weeks 2 to 4 that remained unchanged to week 6, with transient increases in CRH mRNA from week 2-4 in the PVN [97]. In addition, 19 days of treadmill exercise was also found to modulate chronic corticosterone administration induced HPA axis hypoactivity [98]. It should be noted that the potential stress involved in forced treadmill training, which is in addition to the physiological effects of exercise stress, might confound these results. Thus, the inclusion of a voluntary exercise group as a control in these experiments would aid in elucidating the direct physiological effects of exercise versus those caused by psychological stress.

HPA axis activation in response to exercise occurs in both male and female rodents, but in females this varies in relation to the oestrus cycle $[86,99]$. To the authors knowledge there were no papers returned from our searches that investigated differences between male and female chronic VWR induced hypothalamic markers of HPA activation. Factors such as CRH or CRH receptor adaptations with VWR, the acute effects of VWR on female hypothalamic HPA activation at different stages of the oestrus cycle, and the effects of exercise on these factors in the context of stress remain unexamined. These are highly noteworthy limitations of the literature at present, given that the prevalence of depression has consistently been demonstrated to be higher in females than males in humans $[100,101]$, and that $80 \%$ of clinical depression is preceded by chronic psychological stress [102-104]. Furthermore, these findings suggest that the 
controllability of exercise, its frequency, and duration, and the sex of the animal undertaking exercise are all potential factors involved in moderating the effects of exercise on hypothalamic input into the HPA axis. The perception of stress during forced exercise is likely to vary between individuals, whether human or rodent, and add to the physiological stress of exercise. Speculatively speaking, this additional stress might constitute a mechanism whereby forced exercise - or psychologically stressful exercise - could exacerbate clinical symptoms of stress, and stress related conditions such as stress induced depression. The VWR induced effects on hypothalamic HPA axis function in female mice in particular, is a gap in the literature urgently requiring examination by future research.

\section{Exercise-induced adaptations in the basal ganglia}

The basal ganglia includes the striatum, comprised of the putamen, caudate nucleus, and nucleus accumbens, as well as the globus pallidus, the subthalamic nucleus and substantia nigra [105]. These nuclei, and the putamen in particular, have roles in the control of muscle tone control and movement due to the input received from the somatosensory and motor cortices, with output tothe motor areas of the cortex [105]. Dysfunction in these regions can lead to bradykinesia and tremors that can severely limit activities of daily living as occurs in Parkinson's disease.

Clinical studies investigating the effects of exercise for the treatment of Parkinson's disease have found task based exercise can aid in improving functional mobility [106], although the mechanisms involved are not well understood. Nevertheless, basic science studies investigating the mechanisms of exercise in the basal ganglia demonstrate changes in oxidative stress markers and antioxidant equilibrium. Moderate treadmill running for 8 weeks increases levels of rodent striatal tyrosine hydroxylase $(\mathrm{TH})$ (an enzyme that catalyses L-tyrosine into dihydroxyphenylalanine or L-DOPA, a dopamine precursor) and returns $\alpha$-synuclein phosphorylation (a protein involved in Lewy body conditions) to close to normal levels [107]. However, this is in contrast to another study, which noted no changes in $\mathrm{TH}$ in the substantia nigra pars compacta with treadmill exercise [108]. It is possible that the forced component of treadmill exercise altered and the mechanisms involved and confounded outcomes, and this highlights the importance of using voluntary exercise methods. TH levels are important because dopamine depletion is a central factor in the aetiology of Parkinson's disease [107]. The potential for TH increases with VWR exercise requires further investigation because it may increase the availability of TH for synthesis into L-DOPA. This has important implications for translation to clinical treatment of Parkinson's disease in humans.
Mixed results are evident about levels of oxidative stress in the basal ganglia in response to exercise. Striatal levels of thiobarbituric acid reactive substances (TBARS), that are involved in cellular oxidative damage, were reduced by treadmill running at 13-17 metres/minute for 3 or 4 days a week [107], but not from exercise for 5 days/week for 8 weeks at $10 \mathrm{~m} / \mathrm{min}, 15 \mathrm{~m} / \mathrm{min}$, or $20 \mathrm{~m} / \mathrm{min}$ [109]. It is noteworthy that treadmill running has been reported to significantly reduce other markers of oxidative damage, such as carbonyl content $[107,110]$, while the antioxidant enzyme superoxidase dismutase (SOD) (an enzyme that catalyses the cellular antioxidant mechanism of superoxide into oxygen and hydrogen peroxide) was found to increase [107]. However, these results also require confirmation with studies using voluntary methods.

Exercise also induces alterations in striatal brain derived neurotrophic factor (BDNF). BDNF is thought to be important for the survival of dopaminergic neurons in the striatum. Thus a lack of BDNF in the striatum has implications for dopamine transmission, as well asfor dopamine deficiency related mobility dysfunction conditions such as Parkinson's disease [11,111]. Striatal BDNF mRNA levels are increased significantly $(\mathrm{p}=0.01)$ with 3 weeks of VWR [112]. Moderate to high intensity downhill treadmill running also increases BDNF protein $(\mathrm{p}=0.001)$ [113], although 18 weeks of level treadmill running does not appear to increase BDNF increase [110]. Interestingly, chronic treadmill running also normalises levels of striatal glial fibrillary acidic protein (GFAP) in mouse models of Parkinson's disease [108,114] suggesting that reductions in markers of pathology may also be possible in humans with this condition.

Conversely, high intensity exercise may have detrimental effects in this region. In the striatum, high intensity treadmill exercise disrupts ERK $1 / 2$ and CREB pathways. This was associated with impairments in implicit memory [115]. Similarly, six months of VWR in female Long-Evans hooded rats significantly increased COX activity in the dorsolateral caudate putamen $(\mathrm{p}<0.01)$ [116]. These findings are consistent with recent systematic review findings suggesting that higher intensity exercise may be detrimental to anti-oxidative capacity in humans [13]. However, high intensity treadmill exercise also increases striatal D2 receptor levels, prevents dopamine transporter protein down regulation [117] and reduces pathological glutamatergic neuroexciteability in the striatum [118]. In addition, moderate chronic treadmill running increases striatal nitrergic nitric oxide synthase (NOS) reactivity suggesting up-regulation of the striatal nitrergic system [119]. This is noteworthy because NOS are signalling molecules implicated in synaptic plasticity that are diminished in degenerative diseases. Overall then, clarity about the benefits versus risks of high intensity exercise in the striatum remains unresolved. 


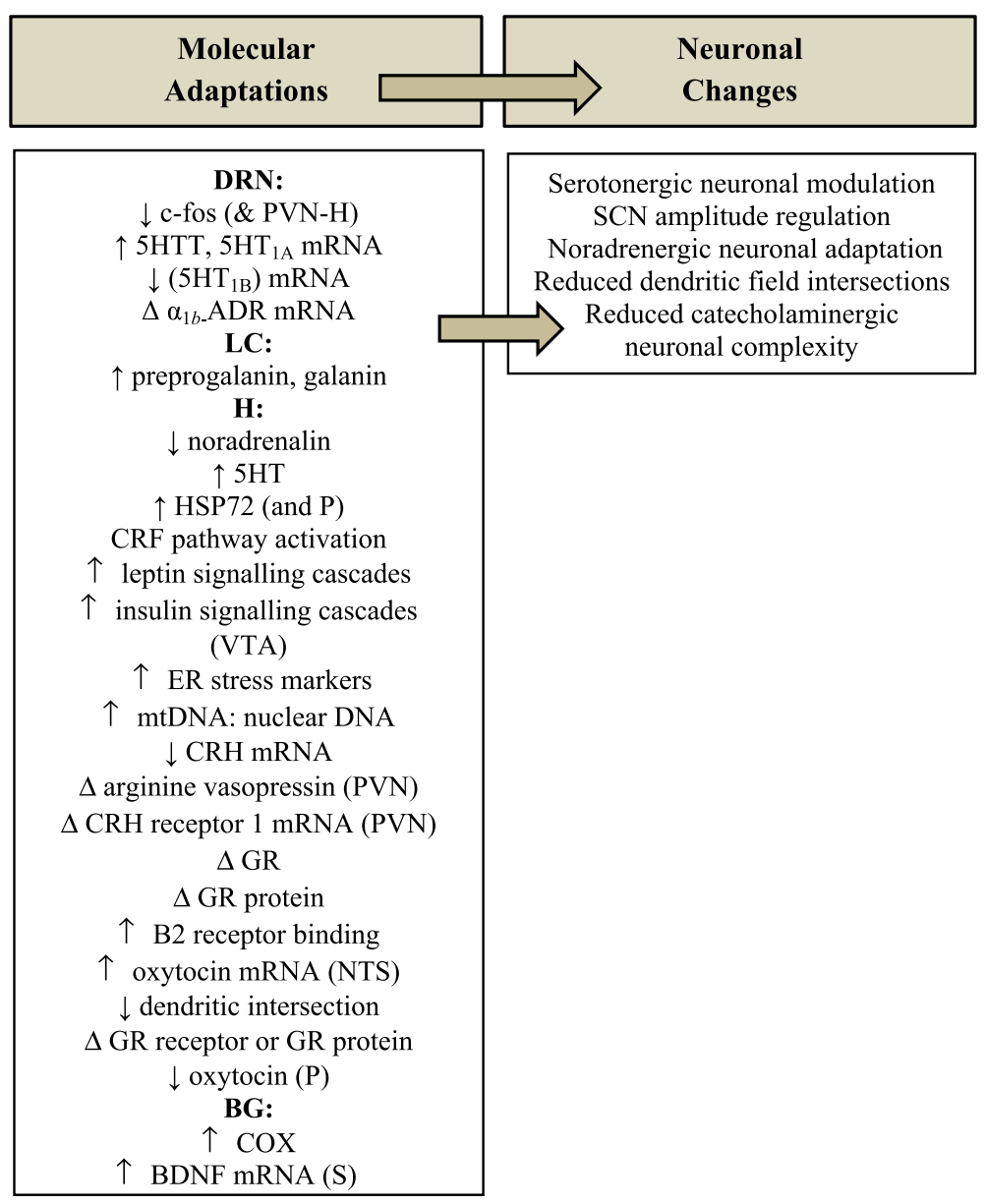

Figure 2 Effects of voluntary exercise in the brain stem, hypothalamus, and basal ganglia. Legend: $\Delta=$ no change; BDNF mRNA = brain derived neurotrophic factor mRNA; c-fos = protein induced acutely by several factors including cytokines; $\mathrm{COX}=$ cytochrome oxidase, an indicator of brain regional functional activity; $\mathrm{CRF}=$ corticotropin releasing factor/hormone; $5 \mathrm{HT}=$ serotonin; $5 \mathrm{HTT}=$ serotonin transporter; $5 \mathrm{HT} 1 \mathrm{~A}$ mRNA = serotonin receptor 1A mRNA; 5HT1B mRNA = serotonin receptor 1B mRNA; $\triangle$ a1b-ADR mRNA = a1b-adrenergic receptor (a1b-ADR) mRNA; $a$-synuclein = precursor protein of amyloid; $\mathrm{DRN}=$ dorsal raphe nucleus; $\mathrm{ER}=$ endoplasmic reticulum; galanin = a regulatory peptide cleaved from preprogalanin;

$\mathrm{GR}=$ glucocorticoid receptor; $\mathrm{H}$ = hypothalamus; $\mathrm{HSP72}=$ heat shock protein 72; mtDNA: nuclear DNA = mitochondrial DNA to nuclear DNA ratio; NOS = nitric oxide synthase; NTS = nucleus tractus solitarii; $\mathrm{P}=$ pituitary; preprogalanin $=$ a precursor of galanin; $\mathrm{PVN}=$ paraventricular nucleus; $\mathrm{BG}=$ basal ganglia; $\mathrm{S}=$ striatum; VTA = ventral tegmental area.

\section{Limitations of the review}

To the author's knowledge, this review constitutes the first brain region specific examination of the neurobiological effects of exercise. Moreover, the review has focussed on CNS functions that become dysfunctional in prevalent conditions such as depression, Parkinson's disease and Alzheimer's disease, factors that are therefore highly pertinent in the current context of globally ageing populations and projected increases in these conditions. However, although this region specific approach provides a novel and worthwhile insight into exercise neuroscience, it does involve some limitations. The examination of other important brain regions, including the limbic system and its interactions on the CNS functions presented herein are complex, and require in depth investigation. Unfortunately, limitations of space preclude such investigations in the present review. Another possible limitation of this review may be that the inclusion of only English published articles could contribute to some selection bias in the results of the review.

\section{Conclusions}

Considerable research has now focussed on the effects of exercise in clinical populations and higher brain regions such as the hippocampus, resulting in greater knowledge about how exercise might support cognitive functioning. However, there appears to be relatively little literature on the effects of exercise on critical centrally mediated mechanisms that involve the functioning of more primitive brain regions.

Nevertheless, this paper has reviewed murine studies examining the effects of exercise on the brain stem, 
hypothalamus, and basal ganglia that constitute basic CNS functions that are critical for health. Important functions of these regions include the circadian clock; energy balance and metabolism; responses to stress and HPA axis functioning; and the maintenance of normal mobility. The functioning of these systems within normal physiological ranges promotes health. Importantly, the dysfunction of these systems is increasingly considered involved in the pathogenesis of a range of prevalent conditions such as depression, Alzheimer's disease, and Parkinson's disease.

The findings reviewed indicate that exercise induces numerous molecular and neuronal adaptations in the brain stem, hypothalamus and basal ganglia. However, a proportion of this work involves forced methods that may differentially affect neurophysiological mechanisms due to the potential for physiological cascades in response to the psychological stress involved in forced exercise. This can confound results [12] leading to misleading findings. Running at intensities greater than are physiologically established by the animal could have adverse effects in some instances [115], and has the added problem of difficulty in the translation to human contexts. In contrast, studies using voluntary wheel running methods have identified a range of regional exercise-induced molecular neurophysiological mechanisms that may contribute to desirable changes in brain region specific functions (see Figure 2).

Voluntary exercise-induced mechanisms mediating stress responsivity in the DRN include serotonergic and adrenergic modulation [32-35] and preprogalanin and galanin in the LC (noradrenergic modulation) [37,40-42]. Hypothalamic metabolic parameters altered by exercise include CRF and leptin signalling modulation [59,60], and changes in food intake [62,63] and markers of MetS [62]. Also in the hypothalamus, exercise-induced increases in B2 receptor bonding sites and dendritic field reductions $[77,79]$ may contribute to altered cardiovascular function. Exercise-induced changes in HPA axis functioning in the hypothalamus appear to be mediated by reduced c-fos expression in the context of exposure to stressors, reduced pituitary oxytocin, and increased HSP72 [52,93]. Finally, in the basal ganglia, voluntary wheel running increases COX activity in the putamen and elevates BDNF mRNA in the striatum $[112,116]$.

Attention to a number of methodological issues by future research will advance the field of exercise neuroscience. First, the forced exercise related findings from all brain regions require replication and confirmation with voluntary wheel running studies. Second, if forced methods are continued, consensus ought to be sought and agreed upon regarding standardised intensities to enable comparable research in the field and the translation to clinical trials. Third, adequately powered studies inclusive of female animals are urgently required to address the gap in the literature about the regional neurobiology of exercise in females. Fourth, future investigated parameters would benefit from the examination of exercise at different ages, to ascertain the effects of exercise throughout the lifespan. This is particularly salient for parameters pertaining to age related conditions such as Parkinson's disease and Alzheimer's disease. By incorporating these considerations into future studies, considerable opportunities to advance exercise neuroscience are available that will result in better understanding of regional brain dysfunctions involved in the aetiology and progression of conditions such as depression, Alzheimer's disease, Parkinson's disease, and many others.

\section{Abbreviations}

CNS: Central nervous system; HPA: Hypothalamic pituitary axis; WWR: Voluntary wheel running; DRN: Dorsal raphe nucleus; RVLM: Rostral ventrolateral medulla; GABA: Gamma-aminobutyric acid; LC: Locus coeruleus; SCN: Suprachiasmic nucleus; HSP72: Heat shock protein 72; MetS: Metabolic syndrome; CRF: Corticotropin releasing factor; IRS2: Insulin receptor 2; VTA: Ventral tegmental area; NTS: Nucleus tractus solitarii; ERS: Endoplasmic reticulum stress; SNS: Sympathetic nervous system; nNOS: Neural nitric oxide synthase; PVN: Paraventricular nucleus; ACTH: Adrenocorticotrophic hormone; GCs: Glucocorticoids; GR: Glucocorticoid receptor; TH: Tyrosine hydroxylase; TBARS: Thiobarbituric acid reactive substances; SOD: Superoxide dismutase; BDNF: Brain derived neurotrophic factor; NOS: Nitrergic nitric oxide synthase; GFAP: Gial fibrillary acidic protein; MPTP: 1-methyl-4-phenyl-1,2,3,6,tetrahydropyridine.

\section{Competing interests}

We wish to confirm that there are no known conflicts of interest associated with this publication. The authors would like to thank the National Health and Medical Research Council (grant APP 1043771 to BT Baune) for the financial support of this work. Julie Morgan would like to thank the lan Wilson Liberal Research Scholarship for the financial support of this work. We confirm that the manuscript has been read and approved by all named authors and that there are no other persons who satisfied the criteria for authorship but are not listed. We further confirm that the order of authors listed in the manuscript has been approved by all of us.

We confirm that we have given due consideration to the protection of intellectual property associated with this work and that there are no impediments to publication, including the timing of publication, with respect to intellectual property. In so doing we confirm that we have followed the regulations of our institutions concerning intellectual property. We understand that the Corresponding Author is the sole contact for the Editorial process (including Editorial Manager and direct communications with the office). He/she is responsible for communicating with the other authors about progress, submissions of revisions and final approval of proofs. Signed by all authors as follows:

Julie A. Morgan ${ }^{1}$

Frances Corrigan ${ }^{2}$

Bernhard T. Baune

\section{Authors' contributions}

JAM drafted and revised the manuscript, and conceived of the manuscript with BB. JAM, FC, and BTB, provided critical review and editing of the manuscript. All authors read and approved the final manuscript.

\section{Authors' information}

JAM; BPhysio; BHealthSci (Hons); SpCertClinRes (Neuroscience). FC; PhD.

BTB; MD, PhD, MPH, FRANZCP.

\section{Author details}

'University of Adelaide, School of Medicine, Discipline of Psychiatry, Psychiatric Neuroscience Laboratory, Adelaide, South Australia, Australia. ${ }^{2}$ University of Adelaide, Discipline of Anatomy and Pathology, School of Medical Sciences, Adelaide, South Australia, Australia. 
Received: 3 February 2015 Accepted: 8 April 2015

Published online: 18 April 2015

\section{References}

1. Pêgo J, Sousa J, Almeida O, Sousa N. Stress and the neuroendocrinology of anxiety disorders. In: Stein MB, Steckler T, editors. Behavioral Neurobiology of Anxiety and Its Treatment. Heidelberg: Springer; 2010. p. 97-118.

2. McEwen BS. Protective and damaging effects of stress mediators: central role of the brain. Dialogues Clin Neurosci. 2006;8(4):367.

3. Cotman C, Engesser-Cesar C. Exercise Enhances and Protects Brain Function. Exercise Sport Sci Rev. 2002;30(2):75-9.

4. Solas M, Aisa B, Tordera RM, Mugueta MC, Ramírez MJ. Stress contributes to the development of central insulin resistance during aging: Implications for Alzheimer's disease. Biochim Biophys Acta (BBA) - Mol Basis Dis. 2013;1832(12):2332-9.

5. Coogan AN, Schutová B, Husung S, Furczyk K, Baune BT, Kropp P, et al. The Circadian System in Alzheimer's Disease: Disturbances, Mechanisms, and Opportunities. Biological Psychiatry. 2013;74(5):333-9.

6. Kronfeld-Schor N, Einat H. Circadian rhythms and depression: Human psychopathology and animal models. Neuropharmacology. 2012;62(1):101-14.

7. Craft S. Insulin resistance syndrome and Alzheimer's disease: Age- and obesity-related effects on memory, amyloid, and inflammation. Neurobiol Aging. 2005;26(1, Supplement):65-9.

8. Pasinetti GM, Eberstein JA. Metabolic syndrome and the role of dietary lifestyles in Alzheimer's disease. J Neurochem. 2008;106(4):1503-14.

9. Belvederi Murri M, Pariante C, Mondelli V, Masotti M, Atti AR, Mellacqua Z, et al. HPA axis and aging in depression: Systematic review and meta-analysis. Psychoneuroendocrinology. 2014:41:46-62.

10. Joshi YB, Praticò D. Stress and HPA Axis Dysfunction in Alzheimer's Disease. In: Pratico D, Meccoci P, editors. Studies on Alzheimer's Disease. New York: Springer; 2013. p. 159-65.

11. Cotman C, Berchtold N. Exercise: a behavioral intervention to enhance brain health and plasticity. Trends Neurosci. 2002;25(6):295-301.

12. Dishman RK, Berthoud HR, Booth FW, Cotman CW, Edgerton VR, Fleshner MR, et al. Neurobiology of exercise. Obesity (Silver Spring). 2006;14(3):345-56.

13. Camiletti-Moiron D, Aparicio VA, Aranda P, Radak Z. Does exercise reduce brain oxidative stress? A systematic review. Scand J Med Sci Sports. 2013;23(4):e202-12.

14. Kohman RA, Kohman RA, Bhattacharya TK, Wojcik E, Rhodes JS. Exercise reduces activation of microglia isolated from hippocampus and brain of aged mice. J Neuroinflammation. 2013;10(1):114.

15. Barrientos RM, Frank MG, Crysdale NY, Chapman TR, Ahrendsen JT, Day HE, et al. Little exercise, big effects: reversing aging and infectioninduced memory deficits, and underlying processes. J Neurosci. 2011;31(32):11578-86.

16. Hindin SB, Zelinski EM. Extended practice and aerobic exercise interventions benefit untrained cognitive outcomes in older adults: a meta-analysis. J Am Geriatr Soc. 2012;60(1):136-41.

17. Karr JE, Areshenkoff CN, Rast P, Garcia-Barrera MA. An empirical comparison of the therapeutic benefits of physical exercise and cognitive training on the executive functions of older adults: a meta-analysis of controlled trials. Neuropsychology. 2014;28(6):829-45.

18. Lees C, Hopkins J. Effect of aerobic exercise on cognition, academic achievement, and psychosocial function in children: a systematic review of randomized control trials. Prev Chronic Dis. 2013;10, E174.

19. Dranovsky A, Hen R. Hippocampal neurogenesis: regulation by stress and antidepressants. Biol Psychiatry. 2006;59(12):1136-43.

20. Spalding KL, Bergmann O, Alkass K, Bernard S, Salehpour M, Huttner HB, et al. Dynamics of Hippocampal Neurogenesis in Adult Humans. Cell. 2013;153(6):1219-27.

21. van Praag H, Kempermann G, Gage FH. Running increases cell proliferation and neurogenesis in the adult mouse dentate gyrus. Nat Neurosci. 1999;2(3):266-70.

22. de la Monte SM, Tong M. Brain metabolic dysfunction at the core of Alzheimer's disease. Biochem Pharmacol. 2014;88(4):548-59.

23. Marazziti D, Rutigliano G, Baroni S, Landi P, Dell'osso L. Metabolic syndrome and major depression. CNS Spectr. 2013;19(4):1-12.

24. Moher D, Liberati A, Tetzlaff J, Altman DG. Preferred reporting items for systematic reviews and meta-analyses: the PRISMA statement. Ann Intern Med. 2009;151(4):264-9.
25. De Bono JP, Adlam D, Paterson DJ, Channon KM. Novel quantitative phenotypes of exercise training in mouse models. Am J Physiol Regul Integr Comp Physiol. 2006;290(4):R926-34.

26. Lin TW, Chen SJ, Huang TY, Chang CY, Chuang Jl, Wu FS, et al. Different types of exercise induce differential effects on neuronal adaptations and memory performance. Neurobiol Learn Mem. 2012;97(1):140-7.

27. Hale MC. Functional topography of midbrain and pontine serotonergic systems: implications for synaptic regulation of serotonergic circuits. Psychopharmacology. 2011;213(2/3):243-64.

28. Hensler JG. Serotonergic modulation of the limbic system. Neurosci Biobehav Rev. 2006;30(2):203-14.

29. Franklin TB, Saab BJ, Mansuy IM. Neural mechanisms of stress resilience and vulnerability. Neuron. 2012;75(5):747-61.

30. Greenwood BN, Fleshner M. Exercise, stress resistance, and central serotonergic systems. Exerc Sport Sci Rev. 2011;39(3):140-9.

31. Greenwood BN, Foley TE, Day HEW, Campisi J, Hammack SH, Campeau S, et al. Freewheel Running Prevents Learned Helplessness/Behavioral Depression: Role of Dorsal Raphe Serotonergic Neurons. J Neurosci. 2003;23(7):2889-98.

32. Greenwood BN, Foley TE, Day HE, Burhans D, Brooks L, Campeau S, et al. Wheel running alters serotonin (5-HT) transporter, 5-HT1A, 5-HT1B, and alpha $1 \mathrm{~b}$-adrenergic receptor mRNA in the rat raphe nuclei. Biol Psychiatry. 2005;57(5):559-68.

33. Greenwood BN, Foley TE, Burhans D, Maier SF, Fleshner M. The consequences of uncontrollable stress are sensitive to duration of prior wheel running. Brain Res. 2005;1033(2):164-78.

34. Greenwood BN, Strong PV, Dorey AA, Fleshner M. Therapeutic effects of exercise: wheel running reverses stress-induced interference with shuttle box escape. Behav Neurosci. 2007;121(5):992-1000.

35. Greenwood BN, Spence KG, Crevling DM, Clark PJ, Craig WC, Fleshner M. Exercise-induced stress resistance is independent of exercise controllability and the medial prefrontal cortex. Eur J Neurosci. 2013;37(3):469-78.

36. Aston-Jones G, Rajkowski J, Cohen J. Role of locus coeruleus in attention and behavioral flexibility. Biol Psychiatry. 1999;46(9):1309-20.

37. Holmes PV, Yoo HS, Dishman RK. Voluntary exercise and clomipramine treatment elevate prepro-galanin mRNA levels in the locus coeruleus in rats. Neurosci Lett. 2006;408(1):1-4.

38. O'Neal HA, Van Hoomissen JD, Holmes PV, Dishman RK. Prepro-galanin messenger RNA levels are increased in rat locus coeruleus after treadmill exercise training. Neurosci Lett. 2001;299(1):69-72.

39. Soares J, Holmes PV, Renner KJ, Edwards GL, Bunnell BN, Dishman RK. Brain noradrenergic responses to footshock after chronic activity-wheel running. Behav Neurosci. 1999;113(3):558-66.

40. Van Hoomissen JD, Holmes PV, Zellner AS, Poudevigne A, Dishman RK, et al. Effects of beta-adrenoreceptor blockade during chronic exercise on contextual fear conditioning and mRNA for galanin and brain-derived neurotrophic factor. Behav Neurosci. 2004;118(6):1378-90.

41. Sciolino NR, Dishman RK, Holmes PV. Voluntary exercise offers anxiolytic potential and amplifies galanin gene expression in the locus coeruleus of the rat. Behav Brain Res. 2012;233(1):191-200.

42. Murray PS, Groves JL, Pettett BJ, Britton SL, Koch LG, Dishman RK, et al. Locus coeruleus galanin expression is enhanced after exercise in rats selectively bred for high capacity for aerobic activity. Peptides. 2010;31(12):2264-8.

43. Ziegler DR, Cass WA, Herman JP. Excitatory influence of the locus coeruleus in hypothalamic-pituitary-adrenocortical axis responses to stress. J Neuroendocrinol. 1999;11(5):361-9.

44. Epp RA, Susser SE, Morissettee MP, Kehler DS, Jassal DS, Duhamel TA. Exercise training prevents the development of cardiac dysfunction in the low-dose streptozotocin diabetic rats fed a high-fat diet. Can J Physiol Pharmacol. 2013;91(1):80-9.

45. Chrousos GP. Stress and disorders of the stress system. Nat Rev Endocrinol. 2009;5(7):374-81.

46. Kondratova AA, Kondratov RV. The circadian clock and pathology of the ageing brain. Nat Rev Neurosci. 2012;13(5):325-35.

47. van Oosterhout F, Lucassen EA, Houben T, vanderLeest HT, Antle MC, Meijer $\mathrm{JH}$. Amplitude of the SCN clock enhanced by the behavioral activity rhythm. PLoS One. 2012;7(6), e39693.

48. Hughes AT, Piggins HD. Feedback actions of locomotor activity to the circadian clock. Prog Brain Res. 2012;199:305-36.

49. Edgar DM, Reid MS, Dement WC. Serotonergic afferents mediate activitydependent entrainment of the mouse circadian clock. Am J Physiol. 1997;273(1 Pt 2):R265-9. 
50. Leise $\mathrm{TL}$, Harrington ME, Molyneux PC, Song I, Queenan H, Zimmerman E, et al. Voluntary exercise can strengthen the circadian system in aged mice. Age. 2013;35(6):2137-52.

51. Didelot C, Schmitt E, Brunet M, Maingret M, Parcellier A, Garrido C. Molecular chaperones in health and disease. In: Starke Br K, Matthias G, editors. Handbook of Experimental Pharmacology, vol. 172. Berlin Heidelburg: Springer; 2006.

52. Nickerson M, Elphick GF, Campisi J, Greenwood BN, Fleshner M. Physical activity alters the brain $\mathrm{Hsp} 72$ and IL-1(beta) responses to peripheral E. coli challenge. Am J Physiol Regul Integr Comp Physiol. 2005;289(6 58-6):R1665-74.

53. Alvaro PK, Roberts RM, Harris JK. A Systematic Review Assessing Bidirectionality between Sleep Disturbances, Anxiety, and Depression. Sleep. 2013;36(7):1059-68.

54. Mattson MP. Energy intake and exercise as determinants of brain health and vulnerability to injury and disease. Cell Metab. 2012;16(6):706-22.

55. Mattson MP. Interventions that improve body and brain bioenergetics for Parkinson's disease risk reduction and therapy. J Parkinsons Dis. 2014;4(1):1-13.

56. Broberger $C$. Brain regulation of food intake and appetite: molecules and networks. J Intern Med. 2005;258(4):301-27.

57. Coppari R, Ichinose M, Lee CE, Pullen AE, Kenny CD, McGovern RA, et al. The hypothalamic arcuate nucleus: a key site for mediating leptin's effects on glucose homeostasis and locomotor activity. Cell Metab. 2005;1(1):63-72.

58. Park S, Jang JS, Jun DW, Hong SM. Exercise Enhances Insulin and Leptin Signaling in the Cerebral Cortex and Hypothalamus during DexamethasoneInduced Stress in Diabetic Rats. Neuroendocrinology. 2005;82(5-6):282-93.

59. Kawaguchi M, Scott KA, Moran TH, Bi S, et al. Dorsomedial hypothalamic corticotropin-releasing factor mediation of exercise-induced anorexia. Am J Physiol Regul Integr Comp Physiol. 2005;288(6):R1800-5.

60. Scarpace PJ, Matheny M, Zhang Y. Wheel running eliminates high-fat preference and enhances leptin signaling in the ventral tegmental area. Physiol Behav. 2010;100(2):173-9.

61. Matheny M, Zhang Y, Shapiro A, Tuemer N, Scarpace PJ. Central overexpression of leptin antagonist reduces wheel running and underscores importance of endogenous leptin receptor activity in energy homeostasis. Am J Physiol Regul Integr Comp Physiol. 2009;297(5):R1254-61.

62. Wagener A, Schmitt AO, Brockmann GA. Early and Late Onset of Voluntary Exercise Have Differential Effects on the Metabolic Syndrome in an Obese Mouse Model. Exp Clin Endocrinol Diabetes. 2012;120(10):591-7.

63. Shapiro A et al. The act of voluntary wheel running reverses dietary hyperphagia and increases leptin signaling in ventral tegmental area of aged obese rats. Gerontology. 2011;57(4):335-42.

64. Borg ML, Andrews ZB, Watt MJ. Exercise training does not enhance hypothalamic responsiveness to leptin or ghrelin in male mice. J Neuroendocrinol. 2014;26(2):68-79.

65. He C, Bassik MC, Moresi V, Sun K, Wei Y, Zou Z, et al. Exercise-induced BCL2regulated autophagy is required for muscle glucose homeostasis. Nature. 2012;481(7382):511-5.

66. Levine B, Kroemer G. Autophagy in the Pathogenesis of Disease. Cell. 2008;132(1):27-42.

67. He C, Sumpter Jr R, Levine B. Exercise induces autophagy in peripheral tissues and in the brain. Autophagy. 2012;8(10):1548-51.

68. Alirezaei M, Kemball CC, Flynn CT, Wood MR, Whitton L, Kiosses WB. Short-term fasting induces profound neuronal autophagy. Autophagy. 2010;6(6):702-10.

69. Özcan U, Cao Q, Yilmaz E, Lee A-H, Iwakoshi NN, Özdelen E, et al. Endoplasmic reticulum stress links obesity, insulin action, and type 2 diabetes. Science. 2004;306(5695):457-61.

70. Kim Y, Park M, Boghossian S, York DA. Three weeks voluntary running wheel exercise increases endoplasmic reticulum stress in the brain of mice. Brain Res. 2010;1317:13-23.

71. Pieczenik SR, Neustadt J. Mitochondrial dysfunction and molecular pathways of disease. Exp Mol Pathol. 2007;83(1):84-92.

72. Marques-Aleixo I, Oliveira PJ, Moreira PI, Magalhães J, Ascensão A. Physical exercise as a possible strategy for brain protection: Evidence from mitochondrial-mediated mechanisms. Prog Neurobiol. 2012;99(2):149-62.

73. Steiner $\mathrm{J}$, Murphy EA, McClellan $J \mathrm{~L}$, Carmichael MD, Davis JM. Exercise training increases mitochondrial biogenesis in the brain. J Appl Physiol. 2011;111(4):1066-71.

74. American College of Sports Medicine. ACSM's guidelines for exercise testing and prescription. 7th ed. China: Lippincott Williams and Wilkins; 2014.

75. Michelini LC, Stern JE. Exercise-induced neuronal plasticity in central autonomic networks: role in cardiovascular control. Exp Physiol. 2009;94(9):947-60.
76. Campbell DJ. The kallikrein-kinin system in humans. Clin Exp Pharmacol Physiol. 2001;28(12):1060-5.

77. Caetano AL, Viel TA, Bittencourt MF, Araujo MS, De Angelis K, Buck HS. Change in central kinin $\mathrm{B} 2$ receptor density after exercise training in rats. Auton Neurosci. 2010;158(1-2):71-8.

78. Martins AS, Crescenzi A, Stern JE, Bordin S, Michelini LC. Hypertension and exercise training differentially affect oxytocin and oxytocin receptor expression in the brain. Hypertension. 2005;46(4):1004-9.

79. Nelson AJ, Juraska JM, Ragan BG, Iwamoto GA. Effects of exercise training on dendritic morphology in the cardiorespiratory and locomotor centers of the mature rat brain. J Appl Physiol. 2010;108(6):1582-90.

80. Mischel NA, Llewellyn-Smith IJ, Mueller PJ. Physical (in)activity-dependent structural plasticity in bulbospinal catecholaminergic neurons of rat rostral ventrolateral medulla. J Comp Neurol. 2014;522(3):499-513.

81. Hsu YC, Chen HI, Kuo YM, Yu L, Huang TY, Chen SJ, et al. Chronic treadmill running in normotensive rats resets the resting blood pressure to lower levels by upregulating the hypothalamic GABAergic system. J Hypertens. 2011;29(12):2339-48.

82. Mastelari RB, de Souza HC, Lenhard A, de Aguiar Correa FM, Martins-Pinge MC. Glutamatergic neurotransmission in the hypothalamus PVN on heart rate variability in exercise trained rats. Auton Neurosci. 2012;170(1-2):42-7.

83. Mastelari RB, de Souza HC, Lenhard A, de Aguiar Correa FM, Martins-Pinge $M C$, et al. Nitric oxide inhibition in paraventricular nucleus on cardiovascular and autonomic modulation after exercise training in unanesthetized rats. Brain Res. 2011;1375:68-76.

84. de Abreu SB, Lenhard A, Mehanna A, de Souza HC, Correa FM, Hasser EM, et al. Role of paraventricular nucleus in exercise training-induced autonomic modulation in conscious rats. Auton Neurosci. 2009;148(1-2):28-35.

85. Adlam D, De Bono JP, Danson EJ, Zhang MH, Casadei B, Paterson DJ, et al. Telemetric analysis of haemodynamic regulation during voluntary exercise training in mouse models. Exp Physiol. 2011;96(11):1118-28.

86. Stranahan AM, Lee K, Mattson MP. Central mechanisms of HPA axis regulation by voluntary exercise. Neuromolecular Med. 2008;10(2):118-27.

87. Cowen PJ. Not fade away: the HPA axis and depression. Psychol Med. 2010;40(1):1-4.

88. Pariante CM, Lightman SL. The HPA axis in major depression: classical theories and new developments. Trends Neurosci. 2008;31(9):464-8.

89. Droste SK, Gesing A, Ulbricht S, Müller MB, Linthorst AC, Reul JM. Effects of long-term voluntary exercise on the mouse hypothalamic-pituitaryadrenocortical axis. Endocrinology. 2003;144(7):3012-23.

90. Campbell JE, Kiraly MA, Atkinson DJ, D'Souza AM, Vranic M, Riddell MC Regular exercise prevents the development of hyperglucocorticoidemia via adaptations in the brain and adrenal glands in male Zucker diabetic fatty rats. Am J Physiol Regul Integr Comp Physiol. 2010;299(1):R168-76.

91. Fediuc S, Campbell JE, Riddell MC. Effect of voluntary wheel running on circadian corticosterone release and on HPA axis responsiveness to restraint stress in Sprague-Dawley rats. J Appl Physiol (1985). 2006;100(6):1867-75.

92. Dishman RK, Bunnell BN, Youngstedt SD, Yoo HS, Mougey EH, Meyerhoff JL. Activity wheel running blunts increased plasma adrenocorticotrophin (ACTH) after footshock and cage-switch stress. Physiol Behav. 1998;63(5):911-7.

93. Campeau S, Nyhuis TJ, Sasse SK, Kryskow EM, Herlihy L, Masini CV, et al. Hypothalamic pituitary adrenal axis responses to low-intensity stressors are reduced after voluntary wheel running in rats. J Neuroendocrinol. 2010;22(8):872-88.

94. Sasse SK, Greenwood BN, Masini CV, Nyhuis TJ, Fleshner M, Day HE, et al. Chronic voluntary wheel running facilitates corticosterone response habituation to repeated audiogenic stress exposure in male rats. Stress. 2008;11(6):425-37.

95. Masini CV, Nyhuis TJ, Sasse SK, Day HE, Campeau S. Effects of voluntary wheel running on heart rate, body temperature, and locomotor activity in response to acute and repeated stressor exposures in rats. Stress. 2011;14(3):324-34.

96. Sasse SK, Nyhuis TJ, Masini CV, Day HE, Campeau S. Central gene expression changes associated with enhanced neuroendocrine and autonomic response habituation to repeated noise stress after voluntary wheel running in rats. Front Physiol. 2013:4:341.

97. Park E, Chan O, Li Q, Kiraly M, Matthews SG, Vranic M, et al. Changes in basal hypothalamo-pituitary-adrenal activity during exercise training are centrally mediated. Am J Physiol Regul Integr Comp Physiol. 2005;289(5):R1360-71.

98. Kim HG, Lim EY, Jung WR, Shin MK, Ann ES, Kim KL. Effects of treadmill exercise on hypoactivity of the hypothalamo-pituitary-adrenal axis induced by chronic administration of corticosterone in rats. Neurosci Lett. 2008;434(1):46-9. 
99. Ogawa S, Chan J, Gustafsson JA, Korach KS, Pfaff DW. Estrogen increases locomotor activity in mice through estrogen receptor alpha: specificity for the type of activity. Endocrinology. 2003;144:230-9.

100. Gater R, Tansella M, Korten A, Tiemens BG, Mavreas VG, Olatawura MO. Sex differences in the prevalence and detection of depressive and anxiety disorders in general health care settings: Report from the world health organization collaborative study on psychological problems in general health care. Arch Gen Psychiatry. 1998;55(5):405-13.

101. Kessler RC. Epidemiology of women and depression. J Affect Disord. 2003;74(1):5-13.

102. Eyre $H$, Baune B. Neurobiological effects of exercise on stress-induced depression. Aust N Z J Psychiatry. 2011;45:A59-60.

103. Bartolomucci A, Leopardi R. Stress and depression: preclinical research and clinical implications. PLoS One. 2009;4(1), e4265.

104. McEwen BS. Mood disorders and allostatic load. Biol Psychiatry. 2003:54(3):200-7.

105. Nolte J. The human brain: an introduction to its functional anatomy. 6 th ed. Philadelphia: Mosby, Elsevier; 2009.

106. Petzinger GM, Fisher BE, McEwen S, Beeler JA, Walsh JP, Jakowec MW. Exercise-enhanced neuroplasticity targeting motor and cognitive circuitry in Parkinson's disease. Lancet Neurol. 2013;12(7):716-26.

107. Tuon T, Valvassori SS, Lopes-Borges J, Luciano T, Trom CB, Silva LA, et al. Physical training exerts neuroprotective effects in the regulation of neurochemical factors in an animal model of Parkinson's disease. Neuroscience. 2012;227:305-12.

108. Dutra MF, Jaeger M, Ilha J, Kalil-Gaspar PI, Marcuzzo S, Achaval M. Exercise improves motor deficits and alters striatal GFAP expression in a 6-OHDAinduced rat model of Parkinson's disease. Neurol Sci. 2012;33(5):1137-44.

109. Aksu I, Topcu A, Camsari UM, Acikgoz O. Effect of acute and chronic exercise on oxidant-antioxidant equilibrium in rat hippocampus, prefrontal cortex and striatum. Neurosci Lett. 2009;452(3):281-5.

110. Lau YS, Patki G, Das-Panja K, Le WD, Ahmad SO. Neuroprotective effects and mechanisms of exercise in a chronic mouse model of Parkinson's disease with moderate neurodegeneration. Eur J Neurosci. 2011;33(7):1264-74.

111. Howells DW, Porritt MJ, Wong JYF, Batchelor PE, Kalnins R, Hughes AJ, et al. Reduced BDNF mRNA Expression in the Parkinson's Disease Substantia Nigra. Exp Neurol. 2000;166(1):127-35.

112. Van Hoomissen JD, Chambliss HO, Holmes PV, Dishman RK. Effects of chronic exercise and imipramine on mRNA for BDNF after olfactory bulbectomy in rat. Brain Res. 2003;974(1-2):228-35.

113. Aguiar Jr AS, Speck AE, Prediger RD, Kapczinski F, Pinho RA. Downhill training upregulates mice hippocampal and striatal brain-derived neurotrophic factor levels. J Neural Transm. 2008;115(9):1251-5.

114. Al-Jarrah MD, Jamous M. Effect of endurance exercise training on the expression of GFAP, S100B, and NSE in the striatum of chronic/progressive mouse model of Parkinson's disease. NeuroRehabilitation. 2011;28(4):359-63.

115. Aguiar Jr AS, Boemer G, Rial D, Cordova FM, Mancini G, Walz R, et al. Highintensity physical exercise disrupts implicit memory in mice: involvement of the striatal glutathione antioxidant system and intracellular signaling. Neuroscience. 2010;171(4):1216-27.

116. McCloskey DP, Adamo DS, Anderson BJ. Exercise increases metabolic capacity in the motor cortex and striatum, but not in the hippocampus. Brain Res. 2001;891(1-2):168-75.

117. Vuckovic MG, Li Q, Fisher B, Nacca A, Leahy RM, Walsh JP, et al. Exercise elevates dopamine D2 receptor in a mouse model of Parkinson's disease: in vivo imaging with [(1)(8)F]fallypride. Mov Disord. 2010;25(16):2777-84.

118. Petzinger GM, Fisher BE, Van Leeuwen JE, Vukovic M, Akopian G, Meshul CK, et al. Enhancing neuroplasticity in the basal ganglia: the role of exercise in Parkinson's disease. Mov Disord. 2010;25 Suppl 1:S141-5.

119. Pietrelli A, Lopez-Costa JJ, Goni R, Lopez EM, Brusco A, Basso N. Effects of moderate and chronic exercise on the nitrergic system and behavioral parameters in rats. Brain Res. 2011;1389:71-82.

\section{Submit your next manuscript to BioMed Central and take full advantage of:}

- Convenient online submission

- Thorough peer review

- No space constraints or color figure charges

- Immediate publication on acceptance

- Inclusion in PubMed, CAS, Scopus and Google Scholar

- Research which is freely available for redistribution

Submit your manuscript at www.biomedcentral.com/submit 